\title{
ON THE COMPLETENESS OF DUAL FOLIATIONS ON NONNEGATIVELY CURVED SYMMETRIC SPACES
}

\author{
RENATO J.M. E SILVA AND LLOHANN D. SPERANÇA
}

\begin{abstract}
We prove Wilking's Conjecture about the completeness of dual leaves for the case of Riemannian foliations on nonnegatively curved symmetric spaces. Moreover, we conclude that such foliations split as a product of trivial foliations and a foliation with a single dual leaf.
\end{abstract}

\section{INTRODUCTION}

A Singular Riemannian Foliation $\mathcal{F}$ on $M$ is a singular foliation, i.e., a decomposition of $M$ into integral submanifolds of an involutive family of smooth vector fields, such that geodesics emanating perpendicularly to an element of $\mathcal{F}$ stays perpendicular to elements of $\mathcal{F}$. Such elements are called leaves.

Given a singular Riemannian foliation $\mathcal{F}$, the dual leaf at $x \in M$ is the subset:

$L_{x}^{\#}=\{q \in M \mid \exists c:[0,1] \rightarrow M, c(0)=x, c(1)=q, c$ is perpenticular to leaves $\}$.

The set of dual leaves define the dual foliation. These concepts and their foundations were introduced by Wilking [ 8 ] and has been used in different situations in literature (see [1, 3, 6, 7], for instance).

In particular, Wilking proves that the dual foliation is a singular foliation (see [8. Proposition 2.1]), moreover, it is Riemannian if $M$ is complete with nonnegative sectional curvature and dual leaves are complete. This is the case in many interesting situations:

Theorem 1. (Wilking [8, Theorem 3]) Suppose that $M$ is a complete nonnegatively curved manifold with a singular Riemannian Foliation $\mathcal{F}$. Then the dual foliation has intrinsically complete leaves if, in addition, one of the following holds:

(1) $\mathcal{F}$ is given by the orbit decomposition of an isometric group action;

(2) $\mathcal{F}$ is a non-singular foliation and $M$ is compact;

(3) $\mathcal{F}$ is given by the fibers of a Sharafutdinov retraction.

Although Theorem 1 gives many interesting conditions for completeness of dual leaves, [8] conjectures that it should be the general case in nonnegative sectional curvature:

Conjecture 1. (Wilking [8]) Suppose $\mathcal{F}$ is a singular Riemannian Foliation on a complete nonnegatively curved manifold. Then the dual foliation has complete leaves.

In this note we give an affirmative answer for Wilking's Conjecture 8, Conjecture] on the completeness of dual leaves in the case of a nonnegatively curved symmetric spaces: 
Theorem 2. Let $\mathcal{F}$ be a singular Riemannian foliation on $M$, a symmetric space with nonnegative sectional curvature. Then, the dual foliation $\mathcal{F}^{\#}$ has complete leaves. Moreover, $\mathcal{F}$ decomposes as a product $\mathcal{F}_{1} \times \mathcal{F}_{2}$ where $\mathcal{F}_{1}$ has a single dual leaf and $\mathcal{F}_{2}$ consists of a single leaf.

That is, there is a metric decomposition $M=Z \times N$, together with a singular Riemannian foliation $\mathcal{F}_{1}$ on $Z$, satisfying $L^{\#}=Z$, such that

$$
\mathcal{F}=\left\{L \times N \mid L \in \mathcal{F}_{1}\right\} .
$$

The result is new even for foliations on the Euclidean space and has an important application to polar foliations. Indeed, one readily recovers the following result:

Theorem 3. Let $\mathcal{F}$ be a polar foliation on $M$ and $\Sigma \rightarrow M$ a polar section. If the action of the Weyl group on $\Sigma$ splits, then $\mathcal{F}$ splits.

Theorem 3 recovers the results in Ewert [2, Theorem 3], Lytchak [5, Lemma 4.1] and Liu-Radeschi [4, Proposition 3.4]. The proof is a direct application of Theorem 2 together with the arguments in [5] sections 2.5 and 4.2]. We refer to section 4 for details.

To prove Theorem 2, we use Lytchak [5, Proposition 3.1] to decompose $M$ as a metric product $M=Z \times N$, where $Z \times\{n\}$ is a minimal dual leaf, then we study the critical points of the distance function between a slice $\{z\} \times N$ and a fixed leaf.

In section 2 we state some useful results and in section 3 we prove Theorem 2 Section 4 relates Theorem 2 to polar foliations.

\section{Preliminaries}

Our main idea to proof Wilking's conjecture relies on decomposing $M$ as a product manifold, where one of the factors is a dual leaf. To this aim, we recall the following result:

Theorem 4. (Lytchak, 5, Proposition 3.1]) Let $M$ be a symmetric space with nonnegative sectional curvature. If $L^{\#}$ is a dual leaf, then $M$ factors as $M=Z \times N$, where $L^{\#}$ is an open subset of $Z \times\{n\}$, for some $n \in N$.

A direct application ensures completeness of dual leaves with minimal dimension.

Proposition 1. Let $M$ be a symmetric space with nonnegative sectional curvature. If $L^{\#}$ is a dual leaf with minimal dimension, then $L^{\#}$ is complete. Moreover,

$$
M=L^{\#} \times N .
$$

Proof. Suppose that $L^{\#}$ is not complete. Then, Theorem 4 gives us a totally geodesic submanifold with the same dimension as $L^{\#}$ such that $L^{\#} \subsetneq Z$.

By hypothesis, the topological boundary of $L^{\#}$ on $Z$ is not empty, on the other hand $b d\left(L^{\#}\right)=\bigcup F^{\#} \subset Z$ is a disjoint union of dual leaves (see Wilking [8, page 1312]). Moreover, since $F^{\#} \subseteq Z$ and $\operatorname{dim} L^{\#}$ has minimal dimension among dual leaves,

$$
\operatorname{dim} L^{\#} \leq \operatorname{dim} F^{\#} \leq \operatorname{dim} Z=\operatorname{dim} L^{\#} .
$$

Therefore, applying Theorem 4 again, each $F^{\#}$ is an open subset of $Z$.

We conclude that the closure of $L^{\#}, L^{\#} \cup b d\left(L^{\#}\right)$, is covered by non-trivial disjoint open subsets. On the other hand, $L^{\#} \cup b d\left(L^{\#}\right)$ is a closed connected subset of $Z$, since $L^{\#}$ is connected, a contradiction. 


\section{Proof of Theorem 2}

We begin the proof by using Proposition 1 to construct very particular vertical vectors outside a minimal dual leaf. We denote by $V$ and $H$ the vertical and horizontal spaces, that is, the space tangent to the leaves and the space orthogonal to $V$, respectively.

Let $L^{\#}=Z$ and $M=Z \times N$ be a fixed closed dual leaf and its respective metric decomposition given by Proposition 1 Fix $(z, n) \in Z \times N$ such that $L^{\#}=Z \times\{n\}$. Denote $Z \times\{n\}=Z_{n}$ and $\{z\} \times N=N_{z}$. The main idea is to use Lemma 1 to show that $N_{z}$ is included in a single leaf, for every $z$.

Let $U$ be a tubular neighborhood of $Z_{n}$ where the square of the distance function $f: U \rightarrow \mathbb{R}$,

$$
f\left(z^{\prime}, n^{\prime}\right)=d_{M}\left(\left(z^{\prime}, n^{\prime}\right), Z_{n}\right)^{2}=d_{N}\left(n^{\prime}, n\right)^{2},
$$

is smooth. Note that the neighborhood $U$ can be chosen as $Z \times B_{n}(r)$, where $B_{n}(r)$ is a convex radius $r$ open ball around $n \in N$ and $r$ does not depend on $n$, since the injectivity radius on symmetric spaces does not depend on the point.

Lemma 1. For every $\left(z^{\prime}, n^{\prime}\right) \in U-Z_{n}$, there exists $v \in T_{\left(z^{\prime}, n^{\prime}\right)} N_{z^{\prime}} \cap V_{\left(z^{\prime}, n^{\prime}\right)}$ such that

$$
\langle v, \nabla f\rangle<0
$$

Proof. We claim that

$$
\nabla f \notin \tilde{H}_{\left(z^{\prime}, n^{\prime}\right)}=\operatorname{pr}_{T N} H_{\left(z^{\prime}, n^{\prime}\right)},
$$

where the right-hand-side is the orthogonal projection of $H_{\left(z^{\prime}, n^{\prime}\right)}$ in $T_{\left(z^{\prime}, n^{\prime}\right)} N$. Recall that $\nabla f\left(z^{\prime}, n^{\prime}\right)$ is the vector in $N_{z^{\prime}}$ defined as the velocity of a minimizing geodesic connecting $\left(z^{\prime}, n^{\prime}\right)$ to $\left(z^{\prime}, n\right)$. Observing that geodesics in $M=Z \times N$ are product geodesics, we conclude that no horizontal vector can be of the form $X+\nabla f, X$ tangent to $Z_{n^{\prime}}$, otherwise there would be a horizontal geodesic, defined by $X+$ $\nabla f$, connecting the dual leaf passing through $\left(z^{\prime}, n^{\prime}\right) \notin Z_{n}$ to the dual leaf $Z_{n}$, a contradiction.

We conclude that $\nabla f \notin \tilde{H}$, thus there exists $v \in \tilde{H}^{\perp} \cap T N_{z^{\prime}}=H^{\perp} \cap T N_{z^{\prime}}=$ $V \cap T N_{z^{\prime}} \neq 0$ such that $\langle v, \nabla f\rangle<0$.

Let $\left(z^{\prime}, n\right) \in Z \times N$ be a point whose leaf we denote by $L_{\left(z^{\prime}, n\right)}$. Define $g_{z^{\prime}}$ : $L_{\left(z^{\prime}, n\right)} \cap U \rightarrow \mathbb{R}$ by

$$
g_{z^{\prime}}(x)=d_{M}\left(x, N_{z}\right)^{2}=d_{M}\left(x, N_{z} \cap U\right)^{2} .
$$

Lemma 2. For every $\left(z^{\prime}, n\right) \in Z_{n}-L_{(z, n)}$,

$$
d_{M}\left(L_{\left(z^{\prime}, n\right)} \cap U, N_{z} \cap U\right)^{2}=\min g_{z^{\prime}}>0 .
$$

Proof. Let $m$ be an arbitrary value for $g_{z^{\prime}}$. We claim that $g_{z^{\prime}}^{-1}(m) \cap Z_{n} \neq \emptyset$. Once proved the claim, we have

$$
m=g_{z^{\prime}}\left(z^{\prime \prime}, n\right)=d_{Z}\left(z^{\prime \prime}, z\right)^{2}>0,
$$

for any given $\left(z^{\prime \prime}, n\right) \in g_{z^{\prime}}^{-1}(m) \cap Z_{n}$. The inequality follows since $z^{\prime \prime} \neq z$ by hypothesis, which completes the proof.

It is sufficient to prove the claim for regular values, since $g_{z^{\prime}}^{-1}\left(\left[m, m^{\prime}\right]\right)$ is closed for every $m^{\prime}>m$ and Sard's Theorem guarantees that the subset of regular values is dense in this interval. 
Let $\epsilon^{2}>0$ be a regular value and denote

$$
S_{z}(\epsilon)=\left\{z^{\prime \prime} \in Z \mid d_{Z}\left(z, z^{\prime \prime}\right)=\epsilon\right\} .
$$

Observe that

$$
g_{z^{\prime}}^{-1}\left(\epsilon^{2}\right)=L_{\left(z^{\prime}, n\right)} \cap U \cap\left(S_{z}(\epsilon) \times N\right) .
$$

Its tangent space satisfy

$$
T g_{z^{\prime}}^{-1}\left(\epsilon^{2}\right)=T L_{\left(z^{\prime}, n\right)} \cap\left(T S_{z}(\epsilon) \times T N\right) \supseteq T L_{\left(z^{\prime}, n\right)} \cap T S(\epsilon)+T L_{\left(z^{\prime}, n\right)} \cap T N .
$$

Because of the last factor, it follows that each point of $g_{z^{\prime}}^{-1}\left(\epsilon^{2}\right)-Z_{n}$ has a vector $v$ as in Lemma 1. Therefore no critical point of $\left.f\right|_{g^{-1}\left(\epsilon^{2}\right)}$ can happen outside $g_{z^{\prime}}^{-1}\left(\epsilon^{2}\right) \cap$ $Z_{n}$. However, supposing $g^{-1}\left(\epsilon^{2}\right) \neq \emptyset,\left.f\right|_{g^{-1}\left(\epsilon^{2}\right)}$ must have a minimum. Since this minimum must happen in $Z_{n}$, we conclude that $g^{-1}\left(\epsilon^{2}\right) \cap Z_{n} \neq \emptyset$ whenever $g^{-1}\left(\epsilon^{2}\right) \neq \emptyset$, completing the proof.

Proof of Theorem Q Since $z, z^{\prime}$ in Lemma 2 are arbitrary, we conclude that $N_{z} \cap$ $U \subseteq L_{(z, n)}$ for every $z$ (equivalently, $N_{z} \cap U \cap L_{\left(z^{\prime}, n\right)}=\emptyset$ whenever $\left(z^{\prime}, n\right) \notin$ $\left.L_{(z, n)}\right)$, thus concluding that $\left.T N_{z}\right|_{U}$ is vertical. In particular, for every $\left(z^{\prime \prime}, n^{\prime \prime}\right) \in$ $U, H_{\left(z^{\prime \prime}, n^{\prime \prime}\right)} \subseteq T Z_{n^{\prime \prime}}$, concluding that $L_{\left(z^{\prime \prime}, n^{\prime \prime}\right)}^{\#}=Z_{n^{\prime \prime}}$ by the minimality of the dimension of $Z$ and Proposition 1. This argument shows that a dual leaf $L_{\left(z^{\prime}, n^{\prime}\right)}^{\#}$ coincides with $Z_{n^{\prime}}$ whenever $n^{\prime}$ is in the tubular neighborhood $U$ of a dual leaf $L_{(z, n)}^{\#}$ that satisfies $L_{(z, n)}^{\#}=Z_{n}$. We conclude the proof by recalling that $U$ can be chosen as $Z \times B_{n}(r)$, where $r$ does not depend on $n$; and that every point $n^{\prime} \in N$ can be connected to a point $n_{0}$, satisfying $L_{\left(z, n_{0}\right)}^{\#}$ has minimal dimension, through a sequence $n_{0}, \ldots, n_{k}$, such that $n_{k}=n^{\prime}$ and $B_{n_{i}}(r) \cap B_{n_{i+1}}(r) \neq \emptyset$.

Now, given a point $z \in Z$, one may ask weather the horizontal space $H_{(z, n)}$, considered as a subspace of $T Z$, vary with $n$ or not. To conclude that it does not vary, consider the parametrized plane $\sigma(s, t)=\left(\gamma_{1}^{t}(s), \gamma_{2}(t)\right)$ defined by (a family of) geodesics $\gamma_{1}^{t} \subset Z, \gamma_{2} \subset N$, such that $\left(\gamma_{1}^{t}\right)^{\prime}(s) \in H$ for every $t, s$. Wilking [8, Proposition 6.1] states that $\sigma$ is a totally geodesic flat, in particular

$$
\frac{\partial \sigma}{\partial s}(s, t)=\left(\gamma_{1}^{t}\right)^{\prime}(s)
$$

is parallel along $t \mapsto \sigma(s, t)$. Thus, by unicity of parallel transport, $\left(\gamma_{1}^{t}\right)^{\prime}(s)=$ $\left(\gamma_{1}^{0}\right)^{\prime}(s)$ for every $s, t$, concluding the statement.

\section{An application to Polar Foliations}

A singular Riemannian foliation is called polar if it admits a totally geodesic horizontal section, i.e., an immersed connected totally geodesic submanifold $\Sigma \rightarrow M$ such that $\Sigma$ intersects every leaf perpendicularly.

One sees that the intersection of $\Sigma$ with the singular strata happens in a set of totally geodesic hypersurfaces of $\Sigma$ and it defines a group of reflections $W$, called the Weyl group. The metric quotient $\Sigma / W$ is isometric to the leaf space $M / \mathcal{F}$.

Now suppose that the action of the Weyl group splits, i.e., $\Sigma=\Sigma_{1} \times \Sigma_{2}$, as a product of groups, and $W=W_{1} \times W_{2}$, as a metric product, such that $W_{i}$ only acts on the $i$-th coordinate. One may ask whether the foliation itself splits. Here we restate and recall the arguments needed to prove Theorem 3 
Theorem 3. Let $\mathcal{F}$ be a polar foliation on $M$ and $\Sigma=\Sigma_{1} \times \Sigma_{2} \rightarrow M$ a polar section. Suppose that the action of the Weyl group splits. Then there is a decomposition $M=M_{1} \times M_{2}$, together with polar foliations $\mathcal{F}_{i}$ on $M_{i}$, such that each leaf of $\mathcal{F}$ is the product of a leaf in $\mathcal{F}_{1}$ and a leaf in $\mathcal{F}_{2}$.

Proof. With Theorem 2 at hand, 3 follows directly from the arguments in [5], section 2.5 and the proof of Proposition 4.2. For convenience, we briefly recall them here.

Suppose that a polar foliation $\mathcal{F}$ is given by a metric quotient $p: M \rightarrow \Delta$, so $\Delta$ is isometric to $\Sigma / W$. Further suppose that $\Sigma$ admits a polar foliation $\mathcal{G}$ which is invariant by $W$ (i.e., $W$ takes $\mathcal{G}$-leaves to $\mathcal{G}$-leaves), thus $W$ acts on $\Sigma / \mathcal{G}=\Delta^{\prime}$. It follows that the fibers of $q \circ p: M \rightarrow \Delta^{\prime}$ defines a polar foliation on $M$ (we refer to [5, section 2.5] for details).

This is certainly the case when the action of $W$ splits. Indeed, denote $q_{i}: \Delta \rightarrow$ $\Delta_{i}=\Sigma_{i} / W_{i}$ the metric quotients. Then $q_{1} \circ p, q_{2} \circ p$ define two polar foliations $\mathcal{F}_{1}^{\prime}$, $\mathcal{F}_{2}^{\prime}$ on $M$, whose sections are $\Sigma_{1}$ and $\Sigma_{2}$, respectively.

By Theorem 2, $M$ decomposes as $M=M_{1} \times M_{2}$, where the leaves of $\mathcal{F}_{1}^{\prime}$ are products of $M_{2}$ with the leaves of a foliation $\mathcal{F}_{1}$ in $M_{1}$. Since every $\mathcal{F}_{1}^{\prime}$-horizontal curve is mapped by $p$ into a $\Delta_{1}$-factor, and hence by $p_{2}$ to a point, any dual leaf to $\mathcal{F}_{1}^{\prime}$ is contained in a $\mathcal{F}_{2}^{\prime}$-leaf. Thus, the $M_{1}$-factor is $\mathcal{F}_{2}^{\prime}$-vertical. The proof is concluded by applying the arguments in the last paragraph of the proof of Theorem 2 to conclude that $\mathcal{F}_{2}^{\prime}$ splits as a foliation $\mathcal{F}_{2}$ in $M_{2}$ and the one-leaf foliation on $M_{1}$. This completes the proof since each leaf in $\mathcal{F}$ is the intersection of a leaf in $\mathcal{F}_{1}^{\prime}$ and one in $\mathcal{F}_{2}^{\prime}$.

\section{ACKNOWLEDGEMENT}

The authors thank A. Lytchak for making them aware of the problem and the application to polar foliations. This work is part of the $\mathrm{PhD}$ thesis of the first author and was partially supported by CNPq [404266/2016-9 to LS]; FAPESP [2017/19657-0 to LS]; and CAPES [88882.329041/2019-01 to RS]

\section{REFERENCES}

1. P. Angulo-Ardoy, L. Guijarro, and G Walschap, Twisted submersions in nonnegative sectional curvature, Archiv der Mathematik 101 (2013), no. 2, 171-180.

2. Heiko Ewert, A splitting theorem for equifocal submanifolds in simply connected compact symmetric spaces, Proceedings of the American Mathematical Society 126 (1998), no. 8, 2443-2452.

3. L. Guijarro and G. Walschap, The dual foliation in open manifolds with nonnegative sectional curvature, Proceedings of the American Mathematical Society 136 (2008), no. 4, 1419-1425.

4. Xiaobo Liu and Marco Radeschi, Polar foliations on symmetric spaces and the mean curvature flow, arXiv preprint arXiv:2006.03945 (2020).

5. A. Lytchak, Polar foliations of symmetric spaces, Geometric and Functional Analysis 24 (2014), no. 4, 1298-1315.

6. L. D. Sperança, On Riemannian foliations over positively curved manifolds, The Journal of Geometric Analysis 28 (2018), no. 3, 2206-2224.

7. L. D. Sperança, Totally geodesic Riemannian foliations on compact lie groups, arXiv:1703.09577, 2017.

8. B. Wilking, A duality theorem for riemannian foliations in nonnegative sectional curvature, Geometric and Functional Analysis 17 (2007), 1297--1320. 
Instituto de Matemática, Estatística e Computação Científica - UniCAMP, Rua Sérgio Buarque de Holanda, 651,13083-97 Campinas - SP, Brazil

E-mail address: renatojuniorms@gmail.com

Instituto de Ciência e Tecnologia - Unifesp, Avenida Cesare Mansueto Giulio Lattes, 1201, 12247-014, SÃo José dos CAmpos, SP, BrazIL

E-mail address: speranca@unifesp.br 